\title{
Prostaglandins, COX-2, and sensory perception
}

\author{
D McCarthy
}

Identification and understanding of the various processes which may underlie the pathogenesis of functional bowel diseases are complex. Apart from the possible contributions of misperception or error, there seem to be four major types of processes potentially involved, namely: (a) motor or secretomotor abnormalities; (b) enteric nervous dysfunction; (c) abnormal sensation; and (d) abnormal central responses to sensory input from peripheral sensations, leading to abnormal responses of efferent autonomic, endocrine, or behavioural activities. In this brief review I will focus on (c) and (d).

Unknown at the present time are: (i) the extent to which visceral pain emanates from hypersensitivity to mechanical, chemical, or other stimuli and (ii) the ways in which learning, conditioning, habituation, long term and short term memory, and explicit or implicit memory or neuronal potentiation, participate in these processes, including the possibility that plastic "memory" (for want of a better word) in peripheral cells and their components (dendrites, nuclei, or axons) or connections (synapses, ganglia, neural networks) is involved in ways in which early or repetitive traumatic life events might act to condition patients to have abnormal sensory responses to normal stimuli at a later stage. My particular inquiries have principally explored the answers to two questions: (1) Are prostaglandins (PGs) involved in these processes? (2) Is $P G$ production from arachidonic acid, the action of cyclooxygenase 2 (COX-2), most relevant to functional bowel disease?

Various types of eicosanoids are made by a variety of cells of the nervous system including neurones (benign and neoplastic) in cell culture, nerve cells in brain, spinal cord, autonomic nerves, and peripheral ganglia, and also in juxtaneuronal cells (astroglia and capillary endothelia) in various sites: products include those of both the cyclooxygenase and lipoxygenase pathways. ${ }^{1}$ While much is known about their localisation, regulation, and functional roles in central sites, less is known about these in spinal, autonomic, and enteric nervous systems but it is now emerging that many mechanisms elucidated centrally may also apply peripherally. ${ }^{1}$

What is known is that there are high concentrations of PGs in all nervous tissue thus far explored. In brain, both COX-1 and COX-2 are expressed constitutively and are probably both inducible, although induction applies mainly to COX-2. In whole brain, there are approximately equal amounts of the messenger RNAs for both COX-1 and COX-2 but the enzymes are found in different loci. COX-1 occurs mainly in forebrain, in areas concerned with consciousness and sensory processing. COX-2 is also expressed and regulated in forebrain (allocortex) but even more so in the hippocampus, amygdala, anteroventral region around the third ventricle, and in discrete brain stem loci. ${ }^{12}$ Exhaustive analysis by Breder et al of the distribution and activation of COX-2 has strongly suggested that PGs derived intracellularly from COX-2, in the absence of any inflammatory processes, are induced physiologically and involved in the activation of autonomic, endocrine, and behavioural responses to visceral and special sensory input. ${ }^{2}$

COX-2 expression in brain, and presumably synthesis of PGs in brain, seems to depend closely on synaptic activity. ${ }^{3}$ Basal levels are higher in awake animals and fall to low levels preceding or during sleep. Much of the activation is dependent on release of glutamate by presynaptic axons. In some synapses, release of an endogenous $N$-methyl-D-aspartate (NMDA) receptor agonist is produced by the action of 5-hydoxytryptamine or opioids on peripheral nerves; such stimuli seem to modify postsynaptic activity-dependent neuroplasticity. Expression of COX-2 is also stimulated by nerve growth factor (NGF) but rapidly reduced by glucocorticoids or deafferentation. NGF increases the incorporation of arachidonic acid into PGs, the rise in PG synthesis always being accompanied by increases in c-fos, c-jun, or other immediate early genes (IEGs) and their products.

The processes of neuronal learning, conditioning, and both explicit and implicit memory must involve neural plasticity (in addition to other processes). The neural changes which contribute to plasticity include elimination of neurones by apoptosis, growth or proliferation of neurones, or of the synaptic connections on or between neurones, alteration in the configuration of neural networks, changes in dominant pathways, or modifications in the group of neurones affected by a particular signal. This fine tuning, based on use, goes on throughout life although it is much greater during development and within the "critical period". Ultimately, the actual structure of neurones must change as their functions change. Exactly how this occurs has not been elucidated but it is clear that PGs are involved. ${ }^{1}$

The sequence of events involves, in brain and spinal cord, release from glutaminergic nerves of neurotransmitter which then binds to NMDA receptors on postsynaptic dendrites, causing calcium to enter cells. This activates phospholipase $\mathrm{A}_{2}$ and releases arachidonic acid

Abbreviations used in this paper: $\mathrm{COX}$ cyclooxygenase; NGF, nerve growth factor; IEGs, immediate early genes; PGs, prostaglandins; NMDA, $\mathrm{N}$-methyl-D-aspartate. 


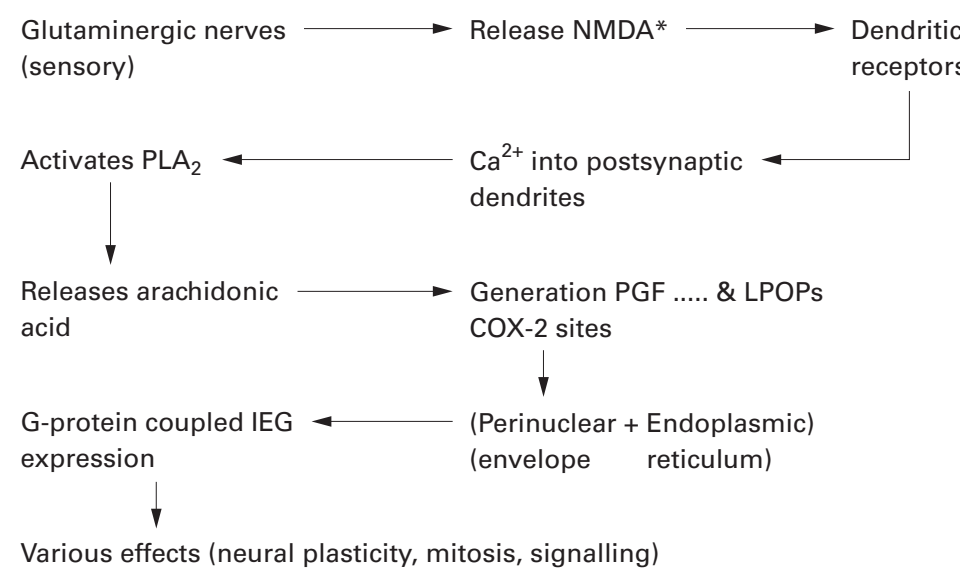

Activity reduced by steroids, deafferentation, non-use

* Precise NMDA receptor agonist(s) unknown

Figure 1 What does cyclooxygenase $2(C O X-2)$ do in the brain? NMDA $N$-methyl-D-aspartate; $P L A_{2}$, phospholipase $A_{2} ; P G F$, prostaglandin $F ; I E G$, immediate early genes; $L P O P$, lipoxygenase products.

substrate for COX-2 and lipoxygenase (fig 1). Arachidonic acid can also be released by the action of phospholipase C. These phospholipases and COX-2 are located in the endoplasmic reticulum and perinuclear envelope, and result in generation of $\mathrm{PG}$ products which then bind to PG receptors within the nucleus, activating a variety of genes, but particularly IEGs such as fos and jun, which are responsive to cyclic nucleotides. Depending on the circumstances, this activation results in a variety of effects such as signalling, mitosis, altered cell shape, apoptosis, etc. Related to neural plasticity, COX-2 products are involved in rapid depolymerisation of actin filaments, followed by a slower repolymerisation that is thought to be stimulated by leukotrienes generated during the same burst of arachidonic acid release: this sequence permits cellular remodelling.

Outside of inflammation, PG production by COX-1 and COX-2 increases rapidly following ischaemia and the products are involved in apoptosis, neuronal elimination, and remodelling of neural connections. COX-2 expression and $P G$ production increase rapidly following convulsions, including electroconvulsive therapy. The PGs produced result in three effects: (1) they are anticonvulsive, (2) they erase memories or change preferred pathways, and (3) they stimulate long term potentiation of newer synaptic connections.

COX-2 expression in brain increases rapidly with stress (for example, cold, swimming, etc) and brief non-injurious electrical stimulation, the effects of brief stresses persisting for long periods (months).

Finally, we come to the possible involvement of PGs, principally of COX-2 origin, in the genesis of pain and perhaps visceral hypersensitivity. These effects of PGs can occur at many sites (table 1). Firstly, they can act peripherally, such as in the gut, where PG and lipoxygenase products such as leukotrienes can stimulate smooth muscle contractions and activate their sensors leading to afferent traffic: this is little studied. In peripheral nerves, PGs sensitise $C$ fibres to painful stimuli. In animal pain models, intravenous administration of antiprostaglandin antibodies which do not cross the blood-brain barrier greatly reduces or abolishes pain behaviour. At the level of the spinal cord, induction of inflammation and pain at a peripheral site (for example, paw) induces spinal activation of NMDA receptors, and release of COX-2 mRNA and PGs at the relevant level of the cord. Exposing the spinal cord to PGs induces hyperalgesia and allodynia. Notably, intrathecal non-steroidal antiinflammatory agents, at doses $<1 \%$ of those needed for a systemic response, abolish the local production of inflammatory mediators and pain in some models. ${ }^{5}$ Finally, many sensory stimuli upregulate COX-2 and COX-1 in various areas of brain known to be involved with pain perception and responses. For all of these reasons, PGs are thought to be important in modifying how pain is perceived and acted upon.

However, recent evidence suggests that the effects of COX-2 on pain may not be mediated solely via PGs, but rather by destruction of endogenous cannabinoids by the enzyme, diminishing the normal analgesic tone in tissues. ${ }^{5}$ These molecules include arachidonyl

Table 1 Prostaglandins and pain — sites of action

- Peripheral sensors? Lipoxygenase products?

- Peripheral nerves:

(i) PGs sensitise $\mathrm{C}$ fibres to analgesia

(ii) Monoclonal AB to PG given iv reduces hyperalgesic behaviour

- Spinal cord

(i) Peripheral inflammation induces NMDA, COX-2 mRNA, PGs and lipoxygenase products by spinal cord at relevant segment level.

(ii) Exposing cord to PGs induces hyperalgesia and allodynia: ?differential nerve effect.

(iii) Intrathecal NSAIDs, at doses $1 \%$ of systemic, abolish inflammatory responses and pain in some models ${ }^{8}$ - Brain: Many stimuli upregulate COX-2 and COX-1, various loci.

PGs, prostaglandins; COX, cyclooxygenase; NMDA, $N$-methyl-D-aspartate.

Table 2 COX-2: role in pain

(1) Glutamate spinal cord hyperalgesia, probably via an increase in COX-2, which then degrades anandamide and PEA below normal tonic analgesic levels.

(2) Blockade of spinal cannabinoid receptors by SR141716A, a $\mathrm{CB}_{1}$ antagonist, increases NMDA dependent hyperalgesia.

(3) This effect is blocked dose dependently by D-AP-5 and MK- 801, two NMDA antagonists.

(4) Selective COX-2 inhibitors may relieve pain by preventing the degradation of endogenous cannabinoids.

COX, cyclooxygenase; NMDA, $N$-methyl-D-aspartate; anandamide, arachidonyl ethanolamide; PEA, palmitylethanolamide. 
ethanolamide and palmitylethanolamide which respectively bind to $\mathrm{CB}-1$ and $\mathrm{CB}-2$ receptors, relieving pain. These appear to be metabolised to PGs by COX-2 but not by COX-1. Intrathecal injection of glutamate leads to hyperalgesia, probably in this way. Intrathecal injection of SR141716A, a CB-1 antagonist, increases NMDA receptor induced hyperalgesia, an effect blocked dose dependently by the NMDA receptor antagonists DAP5 and MK-801. These observations suggest that the analgesic effects of selective COX-2 inhibitor nonsteroidal anti-inflammatory agents may be mediated by maintaining high tissue levels of endogenous cannabinoids involved in the normal damping down of painful stimuli. However, these cannabinoids are degraded in the presence of inflammation, or other inducers of COX-2, leading to pain (table 2). This may be prevented by drugs which inhibit COX-2, regardless of their selectivity. The study of PGs in the enteric nervous system seems to be a fruitful area for further research.

1 Kaufmann WE, Andreasson KI, Isakson PC, et al. Cyclooxygenase and the central nervous system. Prostaglandins 1997;54:601-24

2 Breder CD, DeWitt D, Kraig RF. Characterisation of inducble cyclooxygenase in rat brain. $\mathcal{F}$ Comp Neurol 1995;355: 296-315.

3 Kaufmann WE, Worley PF, Pegg J, et al. P. COX-2, a synaptically induced enzyme, is expressed by excitatory neurons at post synaptic sites in rat cerebral cortex. Proc Natl Acad Sci USA 1996;93:2317-21.

4 Yamagata K, Andreasson KI, Kaufmann WE, et al. Expression of a mitogen-inducible cyclooxygenase in brain neurons. Neuron 1993;11:371-86.

neurons. Neuron 1993;11:371-86.

Calignano A, La Rena G, Graffrida A, et al. Control of pain initiation by endogenous cannabinoids. Nature 1998;394: 277-81.

6 Richardson JD, Aanonsen L, Hargreaves KM. Hypoactivity of the spinal cannabinoid system results in NMDAdependent hyperalgesia. F Neurosci 1998;18:451-7.

7 Portanova JP, Zhang Y, Anderson GD, et al. Selective neutralization of prostaglandin E2 blocks inflammation, hyperalgesia and interleukin-b production in vivo. 7 Exp Med 1996:184:883-91.

8 Malmberg AB, Yaksh TL. Anti-nociceptive actions of spinal non-steroidal anti-inflammatory agents on the formalin test in the rat. F Pharmacol Exp Ther 1992;263:136-45. 